'Programa de Medicina, Facultad de Ciencias de la Salud, Universidad de Boyacá, Tunja, Colombia.

2Programa de Bacteriología, Facultad de Ciencias de la Salud, Universidad de Boyacá, Tunja, Colombia.

${ }^{3}$ Facultad de Medicina, Universidad de La Sabana, Chía, Cundinamarca, Colombia.

${ }^{4}$ Facultad de Medicina, Universidad del Sinú - Elías Bechara Zainúm - Seccional Cartagena, Colombia. aBióloga, MSc., PhD. (Genética humana).

'Bacterióloga, Esp. (Hematología), MgEducación. 'Bacteriólogo, Esp., MSc. (Administración de Salud). 'Médico Genetista, PhD. eQuímica Farmacéutica, Esp., MSc. (Bioquímica).

Este trabajo recibió financiamiento del Proyecto No. 67082 - Convocatoria 844-2019 MinCiencias (Gobierno de Colombia) para su publicación.

Los autores declaran no tener conflictos de interés.

Recibido el 11 de junio de 2020, aceptado el 5 de agosto de 2021.

Correspondencia a: Marcela Díaz Matallana, PhD. mdiazmatallana@gmail.com

\section{Anemia falciforme: una revisión sobre el genotipo de la enfermedad, haplotipos, diagnóstico y estudios asociados}

\author{
MARCELA DÍAZ-MATALLANA ${ }^{1, a}$, \\ YUSSELFY MÁRQUEZ-BENÍTEZ2,b, \\ JULIO CÉSAR MARTÍNEZ-LOZANO ${ }^{3, c}$, \\ IGNACIO BRICEÑO-BALCÁZAR ${ }^{3, \mathrm{~d}}$, \\ ESCILDA BENAVIDES-BENÍTEZ ${ }^{4, e}$, JAIME E. BERNAL ${ }^{4, \mathrm{~d}}$
}

\section{Disease genotype, haplotypes, diagnosis and associated studies in sickle cell anemia}

Sickle cell anemia is a type of hemoglobinopathy characterized by a specific mutation in the beta globin gene with the consequent generation of an unstable hemoglobin that crystallizes in a state of hypoxia. This causes a change in the structure of the red blood cell, which ends up producing vaso-occlusion with the corresponding clinical complications for the patient. Worldwide, various diagnostic tests have been developed that allow the appropriate approach to the affected patient. These include techniques for the determination of hemoglobin and the use of molecular markers, among others. There are new therapeutic alternatives to the use of hydroxyurea and L-glutamine, such as the use of gene therapy tools. The most recent experimental trials are exploring gene editing techniques.

(Rev Med Chile 2021; 149: 1322-1329)

Key words: Anemia, Sickle Cell; Diagnosis; Genetics; Therapeutics.
L a Organización Mundial de la Salud (OMS) considera a la anemia como un problema de interés en salud pública y plantea la meta de reducirla en 50\% para el 2025, especialmente en niños y mujeres en edad reproductiva, población considerada como la más afectada ${ }^{1}$. Dentro de este tipo de patologías, la hemoglobinopatía anemia falciforme $(\mathrm{HbS})$ ha generado gran interés dentro de la comunidad científica por su crecimiento vertiginoso en la población. El número de recién nacidos con anemia falciforme podría pasar de 305.800 en 2010 hasta alrededor de 404.200 en 2050 a nivel global ${ }^{1,2}$.

Las hemoglobinopatías son enfermedades de origen genético, en la estructura o síntesis de la molécula de hemoglobina, la cual está constituida por dos cadenas $\alpha$ y dos cadenas $\beta$, ensambladas con un átomo de hierro que constituye el grupo hemo $^{1-3}$. Dichas cadenas son codificadas por genes independientes localizados en los cromosomas $11 \mathrm{p} 13$ y $11 \mathrm{p} 15.5^{4}$. La hemoglobinopatía anemia falciforme, también conocida como anemia drepanocítica o drepanocitosis (del griego drèpanos $=\mathrm{hoz})^{5-7}$. De acuerdo con la Organización Nacional de Enfermedades Raras - NORD (USA), la frecuencia de anemia falciforme varía entre países ${ }^{6-9}$. Las mutaciones en el gen $H B B$ (complejo globínico Beta) son comunes en personas de África, el Mediterráneo, Medio Oriente, India, en personas del Caribe, regiones de Centro y Suramérica, aunque pueden ser encontradas en individuos de cualquier origen étnico ${ }^{8}$. 
La herencia de la anemia falciforme es de tipo autosómica recesiva, caracterizada por la producción de la hemoglobina S, que difiere de la $\mathrm{HbA}$ normal del adulto, por un cambio de adenina por timina en el gen de la cadena $\beta$ (HBB: c.20 A $>$ T, rs334), resultando en una sustitución del aminoácido ácido glutámico por valina en la sexta posición de la proteína ${ }^{3,10,11}$. Debido a esta mutación, se produce una hemoglobina inestable que se cristaliza fácilmente cuando la presión de oxígeno es baja ${ }^{6}$, lo cual hace que el hematíe se deforme y se torne rígido adoptando la forma de media luna, provocando su destrucción y la consecuente anemia hemolítica ${ }^{12}$.

Todos los individuos con hemoglobina S presentan la misma mutación, la cual va acompañada por otras mutaciones en la región del cromosoma 11, conocidas como grupo de la globina beta ("beta globin cluster"), lo que origina distintos polimorfismos. El patrón de combinación de estos polimorfismos da lugar a los haplotipos que se heredan junto con la mutación de la hemoglobina $S$

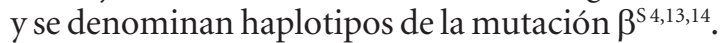
Tales variantes han evolucionado de forma rápida en los últimos 10,000 años ${ }^{6,15}$.

Esta revisión procura dar a conocer avances recientes sobre la anemia falciforme, que indican que esta enfermedad no sólo se ha encontrado en poblaciones de origen africano, sino también se encuentra en otros grupos étnicos. Se mencionan pruebas actuales que permiten un diagnóstico eficaz y en menor tiempo, así como técnicas destinadas al tratamiento, tales como la terapia génica y CRISPR/Cas9, que pretenden mejorar la calidad de vida de los individuos afectados.

\section{Metodología}

Se realizó una búsqueda electrónica en las bases de datos, ScienceDirect, Cochrane, PubMed, con palabras clave como: Anemia falciforme, Genotipo, haplotipos, Biomarcadores, DHPLC, terapia génica, CRISPR/Cas9, y la combinación de ellos que incluyeron artículos de revisión y productos de investigación; además de la utilización de libros electrónicos que apoyaron la fundamentación teórica. Se tuvo en cuenta artículos completos en idioma inglés y español, se realizó la búsqueda de la literatura en los últimos 12 años, es decir con un período de publicabilidad de $80 \%$ no mayor a 10 años y algunos anteriores como referente histórico.

\section{Genotipo de la enfermedad}

La anemia falciforme se presenta como: 1) Forma homocigota (HbSS), o pacientes afectados es decir que manifiestan la enfermedad, considerada como una de las formas más severas de anemia falciforme, la cual se caracteriza por manifestaciones clínicas a partir de dos eventos importantes, la anemia hemolítica y la vaso-oclusión; 2) Forma heterocigota (HbAS) o rasgo drepanocítico, es el paciente asintomático considerado portador sano, pero en condiciones ambientales o fisiológicas de demanda aumentada de oxígeno o hipoxia pueden presentar síntomas. En este, la concentración de $\mathrm{HbS}$ es menor de $50 \%$ y presentan más contenido de $\mathrm{HbA}^{3,16,17}$.

Otros posibles genotipos son: 3 ) Doble heterocigoto $\mathrm{Hb}$-talasemia ( $\mathrm{HbS}$-Tal); en la que se observa la presencia de dos alelos anormales, $\mathrm{HbS}$ y otro para betatalasemia; predominan en el mediterráneo $^{12,18,19}$; 4) Doble heterocigoto $\mathrm{HbS}-\mathrm{HbC}(\mathrm{HbSC})$; en la que se presenta en un alelo niveles de HbS y en el otro alelo niveles de $\mathrm{HbC}^{12,20,21}$.

\section{Herencia de la hemoglobina $S$}

Con respecto a la herencia de la hemoglobina $S$, esta se presenta de las siguientes maneras: 1) Si un progenitor es portador de $\mathrm{Hb} S$ (rasgo falciforme) y el otro no, existe la probabilidad de que el $50 \%$ de los hijos sean portadores y el otro $50 \%$ sanos $^{12}$; 2) Si ambos padres presentan el rasgo falciforme se generarán las siguientes probabilidades: a) $25 \%$ de probabilidad de que el hijo sea sano, es decir herede la hemoglobina normal de ambos progenitores; b) 25\% de probabilidad de que el hijo herede la $\mathrm{Hb} \mathrm{S}$ de ambos padres y por consiguiente correspondería al homocigoto para la enfermedad (HbSS), este hijo presentaría la anemia falciforme ${ }^{22}$; c) $50 \%$ de probabilidad de que el hijo herede la hemoglobina normal de uno de sus padres y la hemoglobina $S$ del otro, es decir, se trataría del heterocigoto $(H B A S)^{18}$.

\section{Rasgo falciforme y la resistencia a la Malaria}

El Plasmodium es un parásito que ha coexistido durante mucho tiempo y ha evolucionado conjuntamente con el huésped humano, esta relación ha ejercido una extraordinaria presión evolutiva sobre el genoma humano. Consecuentemente, 
los seres humanos han seleccionado múltiples polimorfismos genéticos que protegen contra complicaciones graves de la malaria ${ }^{10,23}$.

En el caso del $P$. falciparum, la resistencia que presentan pacientes con rasgo falciforme se deriva de la inhibición de crecimiento del parasito dependiente de la baja de oxígeno, en la cual los parásitos que se encuentran en eritrocitos de pacientes con HbS mantenidos a bajas concentraciones de oxígeno se estancan en una etapa específica en el medio del crecimiento intracelular antes de la replicación del $\mathrm{ADN}^{12,24}$.

\section{Clínica de la enfermedad}

Normalmente, el recién nacido presenta gran cantidad de hemoglobina Fetal, la cual empieza a disminuir hacia los 4 a 6 meses de vida; esta hemoglobina presenta alta afinidad por el oxígeno lo que hace que los pacientes sean asintomáticos al momento del nacimiento ${ }^{25}$. En los pacientes afectados, las manifestaciones clínicas son: la hemólisis marcada, y aquellas que son consecuencia de la vaso-oclusión, generada a partir de la obstrucción de los vasos sanguíneos por la forma de hoz del hematíe; estos episodios terminan por ocasionar daño sistémico en diferentes órganos producto de la isquemia e infartos tisulares ${ }^{12,26-29}$. Otras eventuales manifestaciones clínicas son: la susceptibilidad a infecciones ${ }^{30}$, sindrome torácico agudo ${ }^{31,32}$, miocardiopatía $a^{33}$, infarto esplénico ${ }^{34,35}$. Los síntomas y complicaciones de la enfermedad pueden variar de leves a graves ${ }^{12,36-38}$. La anemia puede ser de tipo normocítica-normocrómica cuando son homocigotos, o, de tipo microcítica mediante el análisis de hemoglobina reticulocitaria ${ }^{39,40}$.

\section{Haplotipos y la clínica de la anemia falciforme}

Los haplotipos se definen como diferentes combinaciones de sitios polimórficos a lo largo de una región cromosómica $(\mathrm{ADN})$, permiten detectar una determinada mutación, cuando ésta se encuentra asociada a un haplotipo particular. En el gen de la hemoglobina beta, estos son segmentos polimórficos a nivel del brazo largo del cromosoma 11 que flanquean al gen a manera de segmentos heterogéneos de ADN. Se han descrito cinco haplotipos principales y varios menores, todos ellos coheredados con la mutación beta $\mathrm{S}$, como alelos sencillos, se ha demostrado su correlación con áreas específicas de África y Asia, y se denominan como haplotipos Benín (Ben), haplo- tipo República Centro Americana (CAR) o Bantú, haplotipo Senegal (Sen), haplotipo Camerún (Cam), haplotipo Arabe-Saudí (Arabe) haplotipo Asiático (Indio o Hindú) y haplotipos menores.

Esta caracterización de haplotipos ha permitido dilucidar mosaicos culturales y étnicos de ciertas poblaciones y aclarar, al menos parcialmente, la razón de la heterogeneidad clínica de la enfermedad, porque dependiendo del haplotipo se pueden presentar cuadros clínicos de anemia falciforme, benigna o severa ${ }^{4,13,15,41}$.

\section{Pruebas diagnósticas: Determinación de hemoglobina}

La técnica de isoelectroenfoque ha sido una prueba bastante utilizada para la detección de hemoglobinopatías, en esta técnica cada hemoglobina tiene un punto isoeléctrico definido ${ }^{42}$. A partir de estas técnicas de electroforesis, es común estimar la prevalencia de la enfermedad ${ }^{3,42}$, así como evaluar la tasa de hemoglobinas anormales en los recién nacidos según el departamento ${ }^{3}$. En el estudio de Rosero y Bermúdez (2012), emplearon la técnica de isoelectroenfoque para analizar hemoglobinopatías a partir de muestras de sangre seca de cordón umbilical ${ }^{3}$.

Se han implementado otras técnicas tales como, la Cromatografía Líquida de Alto Rendimiento Desnaturalizante (DHPLC) $)^{11,43,44}$, así como la electroforesis en gel de agarosa a $\mathrm{pH}$ alcalino ${ }^{4,36}$. Sobre esta última, los autores Romero-Casas et al. (2015) mencionan dos metodologías utilizadas para la evaluación electroforética, como son: 1) Electroforesis en gel de agarosa en medio alcalino SEBIA ${ }^{\oplus}$ para determinación de hemoglobinas, con una corrida en medio ácido SEBIA ${ }^{\circledast}$; 2) Electroforesis capilar en medio acuoso, contenido en un pequeño capilar de sílice que en presencia de corriente eléctrica, permite una separación más rápida con mínimos volúmenes de muestra ${ }^{45}$.

\section{Estudios con marcadores bioquímicos}

Varios estudios evidencian la participación del estrés oxidativo en la fisiopatología de la anemia falciforme. Una excesiva producción de anión superóxido, peróxido de hidrógeno y radical hidroxilo, junto con una menor actividad de las enzimas antioxidantes, conlleva a la producción del daño oxidativo ${ }^{46-48}$.

En una investigación de la Universidad de Granada (España), uno de los autores (IR) eva- 
luó el estado del estrés oxidativo en pacientes panameños a través de las mediciones de siete (7) biomarcadores en el plasma [peroxidación lipídica (LPO), nitritos y nitratos (NOx)]; y en los eritrocitos [Glutatión oxidado (GSSG), glutatión reducido (GSH), glutatión reductasa (GRd), glutatión peroxidasa $(\mathrm{GPx})$, superóxido dismutasa $(\mathrm{SOD})]^{46}$. En general, los resultados evidenciaron que, los pacientes con anemia falciforme exhiben una sobreproducción de radicales libres de oxígeno, que dañan la membrana celular y contribuyen a la hemólisis ${ }^{46}$.

\section{Estudios con marcadores moleculares}

Los haplotipos de la anemia falciforme fueron determinados inicialmente por análisis de fragmentos de longitud polimórfica (RFLPs) en el grupo del gen $H B B^{13}$. A nivel nacional e internacional, se siguen reportando trabajos basados en PCR-RFLPs ${ }^{4,36}$.

Otros autores ${ }^{9,11}$, han analizado esta enfermedad basándose en la técnica de PCR en tiempo real. Estudios más recientes a nivel internacional, han implementado SNPs para la detección de mutaciones puntuales asociadas a la anemia falciforme ${ }^{13-15,37}$. Schriner \& Rotimi (2018), emplearon datos de secuencia de genomas completos a partir del Proyecto 1000 genomas seleccionando cuatro SNPs para su análisis de haplotipos asociados al grupo del gen $\beta$-globina [15], mientras que Shaikho et al. (2017), utilizaron una matriz de datos de SNPs ("SNP array data") escogiendo cuatro SNPs para su análisis de haplotipos asociados al grupo del gen $\beta$-globina ${ }^{13}$. Entre tanto, Fatima \& Mussaed (2015), llevaron a cabo la secuenciación génica y el análisis de un único SNP (rs334) de la hemoglobina $\beta$ involucrada en la anemia falciforme ${ }^{37}$.

\section{Tratamiento}

Tanto la educación como el asesoramiento genético de los pacientes y sus familias, la intervención precoz, así como las pruebas diagnósticas para detectar las complicaciones en el desarrollo temprano se hacen indispensables, de modo que estas puedan tratarse antes de que sean graves, lo cual podrá mejorar significativamente los resul$\operatorname{tados}^{8,10,49}$.

La hidroxicarbamida (hidroxiurea) fue apro- bada por la Administración de Drogas y Alimentos (FDA) para el tratamiento de anemia falciforme y se recomienda para la mayoría de los pacientes con las formas $\mathrm{S} / \mathrm{S}$ y talasemia beta-cero. Se debe ofrecer a los niños con estas formas a los 9 meses de edad ${ }^{8}$. Por otro lado, las transfusiones de sangre pueden usarse en algunos casos: en el caso de una anemia muy grave, en la preparación para una cirugía y para disminuir el riesgo de una crisis severa. En algunas personas, la cirugía puede ser necesaria debido al daño de órganos específicos (vesícula biliar) ${ }^{8}$.

En 2017, la FDA aprobó Endari (L-glutamina) para pacientes de cinco años de edad y mayores con anemia falciforme, para reducir complicaciones graves asociadas con esta afección ${ }^{8,10}$.

En Colombia, mediante la ley 1980 (26/07/2019), se creó el programa de Tamizaje Neonatal ${ }^{50}$, en el cual se amplía el tamizaje neonatal para recién nacidos, para otras enfermedades como: fibrosis quística, fenilcetonuria, galactosemia, hiperplasia suprarrenal congénita, déficit de biotinidasa y hemoglobinopatías, evaluación temprana en busca de sordera y ceguera (33 enfermedades detectadas en total).

Derecho al tamizaje neonatal. A partir de la entrada en vigencia de dicha ley el Ministerio de Salud y Protección Social, a través del Sistema General de Seguridad Social en Salud, garantizará que, de manera progresiva, obligatoria y gratuita, a todo recién nacido vivo se le realice mínimamente un tamizaje neonatal básico, auditivo y visual enmarcado dentro de los lineamientos de salud pública y del modelo de prestación en redes integrales de atención en salud ${ }^{50}$.

\section{Terapia génica}

Según una revisión sistemática realizada por Olowoyeye \& Okwundu $(2012)^{51}$, se llevaron a cabo búsquedas de ensayos experimentales que utilizaran este enfoque de reemplazo de genes defectuosos para obtener hemoglobina con genes normales en el tratamiento de la anemia falciforme. Los autores concluyeron que se requieren más ensayos que evalúen los beneficios o los riesgos de la terapia génica para esta enfermedad ${ }^{51}$.

Una de las terapias es el uso de vectores lentivirales en la cual se extraen células madre hematopoyéticas de la médula ósea del paciente que son cultivadas in vitro y se les introduce copias sanas del gen por medio de un vector lentivirus ${ }^{52}$. Esta 
terapia consiste en transferir un gen de $\beta$-globina bajo el control transcripcional de elementos reguladores del locus de $\beta$-globina. Empero, se están explorando mejoras de vectores para optimizar la seguridad y la eficiencia de estos vectores ${ }^{53}$.

Edición de genes. Recientemente, ha crecido el interés por emplear la técnica CRISPR/cas 9 como procedimiento terapéutico en la corrección del gen $\beta$-globina mutado ${ }^{54,55}$. La edición de la mutación $\beta^{\mathrm{S}}$ por reparación de ADN dirigida por homología ["Homology Directed Repair" (HDR)], se puede conseguir por medio de nucleasas con zinc o CRISPR/Cas9, para escindir el locus $\beta^{\mathrm{S}}$, por otra parte, genomas virales u oligonucleótidos monocatenarios actúan como moldes donantes $\mathrm{HDR}^{56}$. La plataforma CRISPR/Cas9 utiliza una única endonucleasa y un solo ARN guía ( $\mathrm{ARNg}$ ) para inducir la ruptura de ADN (doble cadena) de secuencia específica. Cuando esto acompaña a un molde de reparación, permite reparar el gen mutado $^{56,57}$.

Una estrategia alterna, tiene como objetivo reactivar la síntesis de Hemoglobina fetal ( $\mathrm{HbF})$ en eritrocitos adultos, la cual se enfoca en la regulación negativa de BCL11A, esto permitiría que los síntomas se presenten de forma más leve ${ }^{58}$. No obstante, esta tecnología aún debe demostrar su perfil de eficacia y bioseguridad en un contexto real.

\section{Conclusiones y reflexiones}

En Colombia, hasta el momento se han realizado pruebas para el diagnóstico de las hemoglobinopatías, a partir de técnicas como el isoelectroenfoque, DHPLC, electroforesis en gel de agarosa a $\mathrm{pH}$ alcalino, electroforesis capilar. A nivel molecular, se han reportado estudios principalmente con marcadores RFLPs, para el análisis de los haplotipos clásicos. En Colombia, no se conocen estudios publicados sobre hemoglobinopatías y haplotipos clásicos basados en técnicas de secuenciación de ADN. El conocimiento de la distribución de los afectados y de los portadores (heterocigotos) en Colombia mediante secuenciación de $\mathrm{ADN}$, es primordial, con el fin de proveer servicios preventivos, tratamientos e información para el público, así como para una disposición adecuada de los recursos en salud.

A nivel internacional, se ha reportado el análisis de biomarcadores para evidenciar estrés oxidativo celular en pacientes afectados con anemia falciforme, donde se ha detectado en dichos pacientes una sobreproducción de radicales libres de oxígeno, que contribuyen a la hemólisis. Por tal razón, sería deseable incorporar este tipo de análisis en muestras de pacientes afectados colombianos provenientes de diversas regiones de Colombia, con el objeto de comprender las distintas manifestaciones clínicas de la enfermedad.

La educación, el asesoramiento genético tanto al paciente como a su familia, la intervención anticipada, así como las pruebas para detectar complicaciones en el desarrollo temprano de esta afección, permitirán mejorar significativamente los resultados.

Cabe destacar que, la expedición de la ley 1980 (26/07/2019, Colombia) enmarcada dentro de los lineamientos de salud pública, el derecho al tamizaje neonatal, después de varios años de esfuerzo conjunto con muchas organizaciones, dicha ley beneficiará a muchos pacientes afectados y sus familias.

Por último, tanto la terapia génica como la edición génica abren un panorama promisorio para el tratamiento de esta enfermedad, de hecho, se han logrado grandes avances en estas áreas a una escala internacional. Sin embargo, se necesitarán muchos más ensayos que evalúen beneficios y/o riesgos de la terapia génica para tratar la anemia falciforme.

Agradecimientos: Agradecemos a MinCiencias por su financiamiento, así mismo, a las Universidades de los autores.

\section{Referencias}

1. Acuña C, Cuero K, Espitia K, Rojas R, Torres R. Anemia drepanocítica y situación en Colombia: Revisión. Biociencias 2018; 3: 65-75.

2. Piel F, Hay S, Gupta S, Weatherall D, Williams T. Global Burden of Sickle Cell Anaemia in Children under Five, 2010-2050: Modelling Based on Demographics, Excess Mortality, and Interventions. PLOS Medicine 2013; 10 (7): 1-14.

3. Rosero MJ, Bermúdez AJ. Análisis de hemoglobinopatías en regiones afrocolombianas usando muestras de sangre seca de cordón umbilical. Acta Médica Colombiana 2012; 37 (3): 118-24. 
4. Durán CL, Morales OL, Echeverri SJ, Isaza M. Haplotipos del gen de la globina beta en portadores de hemoglobina S en Colombia. Biomédica 2012; 32: 103-11.

5. Gallori E. Atlas Ilustrado de Genética. Susaeta Ediciones, S.A. Madrid. 2012; 240.

6. Schaefer GB, Thompson Jr JN. Genética Médica, Un Enfoque Integrado. McGraw-Hill Interamericana Editores, S.A. de C.V. México, D.F. 2016; 372.

7. Del Castillo Ruíz V, Uranga Hernández RD, De la Rosa GF. Genética clínica. Editorial El Manual Moderno. 2da edición. México, D.F. 2019; 651.

8. Bender MA. Sickle cell Disease. Department of Pediatrics, University of Washington, Fred Hutchinson Cancer Research Center, Seattle, Washington (USA) 2019. NORD - National Organization for Rare Disorders, Inc. Available from: https://rarediseases.org/rare-diseases/ sickle-cell-disease/

9. Fong C, Menzel S, Lizarralde MA, Barreto G. Genetic variants associated with fetal hemoglobin levels show the diverse ethnic origin in Colombian patients with sickle cell anemia. Biomédica 2015; 35: 437-43.

10. OMIM Database (1966-2019). Sickle Cell Anemia. Johns Hopkins University. Available from: https://www.omim. org/entry/603903?search=sickle\%20cell\%20anemia\&highlight=cell\%20anemia\%20anaemia\%20sickle [02-052019].

11. Yue L, Lin M, Chen JT, Zhan XF, Zhong DS, MonteNguba SM, et al. Rapid screening for sickle cell disease by polymerase chain reaction-high resolution melting analysis. Mol Med Rep 2014; 9: 2479-84.

12. Quintero M, Jiménez Hernández A. Anemia de Celulas Falciformes. Gastrohnup. 2012; 14 (2): s27-35. Available from: http://bibliotecadigital.univalle.edu.co/ bitstream/10893/5929/1/12 Anemia.pdf

13. Shaikho EM, Farrel JJ, Alsultan A, Qutub H, Al-Ali AK, Figuereido MS, et al. A phased SNP-based classification of sickle cell anemia $H B B$ haplotypes. BMC Genomics. 2017; 18 (608): 1-7.

14. dBSNP Short Genetic Variations. National Center of Biotechnology Information (NCBI), U.S. National Library of Medicine. Available from: https:/www.ncbi. nlm.nih.gov/SNP/

15. Shriner D, Rotimi C. Whole-Genome-Sequence-Based Haplotypes Reveal Single Origin of the Sickle Allele during the Holocene Wet Phase. Am. J. Hum. Genet. 2018; 102: 547-56.

16. Agramonte Llanes OM, Expósito Delgado Y, Morales MM, Zamora González Y. ¿Es realmente asintomático el portador de la hemoglobina S? Rev Cuba Hematol Inmunol y Hemoter. 2015; 31 (2): 102-12.
17. Key NS, Derebail VK. Sickle-cell trait: novel clinical significance. Hematol Am Soc Hematol Educ Progr. 2010; 418-22.

18. Masilamani V, Devanesan S, AlQathani F, AlShebly M, Daban HH, Canatan D, et al. A Novel Technique of Spectral Discrimination of Variants of Sickle Cell Anemia. Dis Markers. 2018; 5942368.

19. Indrák K, Divoká M, Pospíšilová D, Čermák J, Beličková M, Horváthová M, et al. Hemoglobinopathies. Vnitr Lek. 2018; 64 (5): 476-87.

20. El Maataoui H, Fahi A, Oukkache B. Sickle cell trait and haemophilia: a rare association. Pan Afr Med J. 2018; 29:92.

21. Sinha MK, Raghuwanshi B, Bag ND, Barman A. Splenic Infarction in Two Members of the Family with Sickle Cell Trait: A Case Report of Rare Complication. Int J Appl basic Med Res. 2017; 7 (4): 272-4.

22. Ehsan M, Maruvada S. Anemia, Sickle Cell [Internet]. StatPearls. StatPearls Publishing. 2018 [cited 2018 Sep 21]. Available from: http://www.ncbi.nlm.nih.gov/pubmed/29489205

23. Waldecker M, Dasanna AK, Lansche C, Linke M, Srismith S, Cyrklaff M, et al. Differential time-dependent volumetric and surface area changes and delayed induction of new permeation pathways in P. falciparum-infected hemoglobinopathic erythrocytes. Cell Microbiol. 2017; 19 (2).

24. Archer NM, Petersen N, Clark MA, Buckee CO, Childs LM, Duraisingh MT. Resistance to Plasmodium falciparum in sickle cell trait erythrocytes is driven by oxygen-dependent growth inhibition. Proc Natl Acad Sci. 2018; 115 (28): 7350-5.

25. Sankaran VG, Orkin SH. The Switch from Fetal to Adult Hemoglobin. Cold Spring Harb Perspect Med. 2013; 3 (1): a011643-a011643.

26. Rojas-Martínez A, Calderón E, Vidal MA, Arroyo F, García-Hernández R, Torres LM. Crisis drepanocítica y tratamiento del dolor. Rev la Soc Española del Dolor. 2015; 22 (4): 165-7.

27. Campelo LMN, Oliveira NF, Magalhães JM, Julião AM de S, Amorim FCM, Coelho MCVS. The pain of children with sickle cell disease: the nursing approach. Rev Bras Enferm. 2018, 71 (suppl 3): 1381-7.

28. Lakkakula BVKS, Sahoo R, Verma H, Lakkakula S. Pain Management Issues as Part of the Comprehensive Care of Patients with Sickle Cell Disease. Pain Manag Nurs. 2018 Jul 31.

29. McGann PT, Nero AC, Ware RE. Clinical Features of $\beta$-Thalassemia and Sickle Cell Disease. Adv Exp Med Biol. 2017; 1013: 1-26.

30. Ayala Viloria AJ, Torres HJG, David Tarud GJ. Anemia 
de células falciformes: una revisión. Salud Uninorte. 2016; 32 (3): 513-27.

31. Wang MX, Pepin EW, Verma N, Mohammed T-L. Manifestations of sickle cell disease on thoracic imaging. Clin Imaging. 2018; 48: 1-6.

32. De Prost N, Sasanelli M, Deux J-F, Habibi A, Razazi K, Galactéros F, et al. Positron Emission Tomography With 18F-Fluorodeoxyglucose in Patients With Sickle Cell Acute Chest Syndrome. Medicine (Baltimore). 2015; 94 (18): e821.

33. Niss O, Quinn CT, Lane A, Daily J, Khoury PR, Bakeer $\mathrm{N}$, et al. Cardiomyopathy With Restrictive Physiology in Sickle Cell Disease. JACC Cardiovasc Imaging. 2016; 9 (3): 243-52.

34. Seegars MB, Brett AS. Splenic infarction associated with sickle cell trait at low altitude. Hematology 2015; 20 (10): 607-9.

35. Goodman J, Hassell K, Irwin D, Witkowski EH, Nuss R. The Splenic Syndrome in Individuals with Sickle Cell Trait. High Alt Med Biol. 2014; 15 (4): 468-71.

36. Aghajani, F, Mahdavi MR, Kosaryan M, Mahdavi M, Hamidi $M$, JalaliH. Identification of $\beta$-globin haplotypes linked to sickle hemoglobin ( $\mathrm{Hb} \mathrm{S}$ ) alleles in Mazandaran province, Iran. Genes Genet. Syst. 2016, 91: 311-3.

37. Fatima SS, Mussaed EM. Gene Sequencing and SNP Analysis of rs334 of Beta Hemoglobin involved in Sickle Cell Anemia. Helix. 2015; 2: 645-7.

38. CDC para el control y prevención de enfermedades. Síntomas y tratamientos | Anemia drepanocítica | NCBDDD | CDC [Internet]. 2017 [cited 2018 Sep 23]. Available from: https://www.cdc.gov/ncbddd/spanish/ sicklecell/symptoms.html

39. Ramírez Cuentas J, Lizama Olaya O, Martínez La Rosa J, Jhong Olivera M, Salazar Lindo E. Reporte de un probable caso de Hemoglobina S / Talasemia Beta. Rev Med Hered [Internet]. 2004 Jul [citado 2020 Abr 15]; 15 (3): 173-8. Disponible en: http:// www.scielo.org.pe/scielo.php?script=sci_arttext\&pi$\mathrm{d}=$ S1018-130X2004000300010\&lng=es.

40. Márquez-Benítez Y, Cruz-Rubio SG, Vargas-Acevedo DM. Hemoglobina de reticulocito y su importancia en el diagnóstico temprano de anemia. Univ. Salud [Internet]. 2018 Dic [citado 2020 Abr 15]; 20 (3): 292-303. Disponible en: http://www.scielo.org.co/scielo.php?script=sci_arttext\&pid=S0124-71072018000300292\&1ng=es. http://dx.doi.org/10.22267/rus.182003.133.

41. Shimizu K, Hashimoto T, Harihara S, Tajima K, Sonoda $\mathrm{S}$, Zaninovic V. Beta-globin gene haplotype characteristics of Colombian Amerindians in South America. Hum Hered. 2012; 51: 54-63.
42. De Bernal M, Collazos A, Bonilla RD, Tascón EP. Determination of the prevalence of hemoglobin S, C, D, and $\mathrm{G}$ in neonates from Buenaventura, Colombia. Colombia Médica 2010; 41 (2): 1-5.

43. Dobrila L, Zhu T, Zamfir D, Tarnawski M, Ciubotariu $\mathrm{R}$, Albano MS, et al. Detection of Hemoglobin ( $\mathrm{Hb}) \mathrm{Va}-$ riants by HPLC Screening in Cord Blood Units (CBU) Donated to the National Cord Blood Program (NCBP). Blood. 2016; 128 (22): 2182.

44. Pregen Colombia. Medicina Genética. 2019. http://www. pregencolombia.com/site/

45. Romero-Sánchez C, Gómez A, Duarte Y, Amazo C, Manosalva C, Chila L, et al. Variantes de hemoglobina en una población con impresión diagnóstica positiva para hemoglobinopatías en Colombia. Rev Med Chile 2015; 143: 1260-8.

46. Rusanova I. Determinación de los haplotipos de anemia falciforme y su correlación con los niveles de estrés oxidativo en pacientes de 6 meses a 15 años de edad en Panamá. Tesis Doctoral, Facultad de Medicina, Depto. Fisiología, Universidad de Granada. Granada, España 2010; 164.

47. Ren H, Chebreneskel K, Okpala I, Lee A, Ibegbulam O, Crawford M. Patients with sickle cell disease have reduced blood antioxidant protection. Int J Vitam Nutr Res. 2008; 78 (3): 139-48.

48. Nur E, Biemond BJ, Otten HM, Brandjes DP, Schnog JJ. CURAMA Study Group. Oxidative stress in sickle cell disease; pathophysiology and potential implications for disease management. Am J Hematol. 2011; 86 (6): 4849.

49. Rigano P, De Franceschi L, Sainati L, Piga A, Piel FB, Cappellini MD, et al. Italian Multicenter Study of Hydroxyurea in Sickle Cell Anemia Investigators. Real-life experience with hydroxyurea in sickle cell disease: A multicenter study in a cohort of patients with heterogeneous descent. Blood Cells Mol Dis. 2018 Mar; 69: 82-9. doi: 10.1016/j.bcmd.2017.08.017. Epub 2017 Oct 9.

50. República de Colombia-Gobierno Nacional (Congreso de la República). Ley 1980 de 26 de Julio de 2019. "Por medio de la cual se crea el programa de Tamizaje Neonatal en Colombia”, Bogotá, D.C.

51. Olowoyeye A, Okwundu CI. Gene therapy for sickle cell disease. Cochrane Database of Systematic Reviews. John Wiley and Sons Ltd. 2012; 1-11.

52. Perumbeti A, Malik P. Genetic correction of sickle cell anemia and beta-thalassemia: progress and new perspective. Scientific World Journal. 2010 Apr 13; 10: 644-54. doi: 10.1100/tsw.2010.67. PMID: 20419277; PMCID: PMC5763711.

53. Cavazzana M, Mavilio F. Gene Therapy for Hemoglo- 
binopathies. Hum Gene Ther. 2018; 29 (10): 1106-13. doi:10.1089/hum.2018.122.

54. Akcakaya P, et al. In vivo CRISPR editing with no detectable genome-wide off-target mutations. Nature. 2018 Sep; 561 (7723): 416-9. doi: 10.1038/s41586-018-0500-9.

55. Hirakawa MP, Krishnakumar R, Timlin JA, Carney JP, Butler KS. Gene editing and CRISPR in the clinic: current and future perspectives. Biosci Rep. 2020 Mar.

56. Kalkan BM, Kala EY, Yuce M, Karadag Alpaslan M, Kocabas F. Development of gene editing strategies for human $\beta$-globin (HBB) gene mutations. Gene. $2020 \mathrm{Apr}$ 15; 734 .

57. Romero Z, Lomova A, Said S, Miggelbrink A, Kuo CY,
Campo-Fernández B, et al. Editing the Sickle Cell Disease Mutation in Human Hematopoietic Stem Cells: Comparison of Endonucleases and Homologous Donor Templates. Mol Ther [Internet]. 2019 Aug 7; 27 (8): 1389-406.

58. Khosravi MA, Abbasalipour M, Concordet JP, Berg J Vom, Zeinali S, Arashkia A, et al. Targeted deletion of BCL11A gene by CRISPR-Cas9 system for fetal hemoglobin reactivation: A promising approach for gene therapy of beta thalassemia disease. Eur J Pharmacol [Internet]. 2019 Jul 5 [cited 2020 Apr 15]; 854: 398405. Available from: http://www.ncbi.nlm.nih.gov/ pubmed/31039344. 